\title{
4-(HIDROXIMETIL)-BENZENOSSULFONATO DE POTÁSSIO: METABÓLITO INÉDITO ISOLADO DA ALGA MARINHA Bostrychia tenella (RHODOMELACEAE, CERAMIALES)
}

Rafael de Felício e Hosana Maria Debonsi*

Departamento de Física e Química, Faculdade de Ciências Farmacêuticas de Ribeirão Preto, Universidade de São Paulo, Av. do Café, s/n, 14040-903 Ribeirão Preto - SP, Brasil

Nair Sumie Yokoya

Seção de Ficologia, Instituto de Botânica de São Paulo, Av. Miguel Stéfano, 3687, 04301-902 São Paulo - SP, Brasil

Recebido em 8/2/08, aceito em 8/4/08, publicado na web em 29/4/08

\begin{abstract}
POTASSIUM 4-(HYDROXYMETHYL)-BENZENOSULFONATE: A NOVEL METABOLITE ISOLATED FROM THE MARINE RED ALGA Bostrychia tenella (RHODOMELACEAE, CERAMIALES). Chemical investigation of the dichloromethane/methanol extract of the marine alga Bostrychia tenella has led to the isolation of two aromatic compounds, the new sulfate metabolite potassium 4-(hydroxymethyl)-benzenosulfonate (1) and the compound 1-methoxyphenethyl alcohol (2), described previously as a synthetic product. Their structures were determined by spectroscopic methods including NMR, MS, IR and by comparison with literature data.
\end{abstract}

Keywords: Bostrychia tenella; natural products; marine red alga.

\section{INTRODUÇÃO}

A intensa investigação por produtos naturais oriundos do reino vegetal resultou em grande evolução na aplicação destes insumos, contribuindo com a melhoria da saúde humana. ${ }^{1}$ Porém, apesar da infinita diversidade vegetal e de todo seu potencial biológico agregado, novas fontes de produtos naturais vêm ganhando destaque nas últimas décadas. ${ }^{2}$ Neste contexto, o oceano - um macro ambiente equilibrado, auto-suficiente e relativamente pouco explorado - causou efeito profundo na química de produtos naturais. Este ambiente tornou-se alvo de pesquisas visando a descoberta de substâncias potencialmente bioativas para fins terapêuticos, dentre outros. ${ }^{3}$ Ainda, deve ser ressaltada a utilização de metabólitos marinhos nas indústrias alimentícia, inseticida e cosmética, o que justifica os esforços contínuos de profissionais nesta nova linha de pesquisa. ${ }^{4}$

Dentre as estruturas químicas diversas sintetizadas por organismos marinhos, não é de se surpreender que haja grande ocorrência de substâncias halogenadas e sulfatadas, uma vez que os íons sulfato, cloreto, brometo e iodeto estão presentes em abundância no ambiente marinho. ${ }^{5}$

Na literatura, são apresentados diversos polissacarídeos sulfatados de importância econômica. São substâncias atóxicas com características gelatinizantes e espessantes que, muitas vezes, demonstram atividade farmacológica. Dentre estes, podemos citar as galactanas encontradas em Bostrychia montagnei, parcialmente sulfatadas e piruvatadas e ainda as agaranas de Acanthophora spicifera, as quais são altamente substituídas por grupos sulfatos e muitas vezes exibem potente e seletiva atividade antiviral contra HSV-1 e HSV-2. ${ }^{6}$ Dentre as substâncias sulfatadas, também estão presentes aquelas não pertencentes à classe dos polissacarídeos, como por exemplo, os raros glicerídeos, ribosídeos, lipídeos e esteróides. Com relação aos compostos fenólicos, há uma produção expressiva de substâncias sulfatadas em gêneros bastante estudados dentro da família Rhodomelaceae, como Polysiphonia ${ }^{7,8}$ e Rhodomela. $^{9-11}$ Substâncias halogenadas, sulfatadas ou não, também são encontradas em núme-

*e-mail: hosana@fcfrp.usp.br ro considerável de espécies das divisões Rhodophyta e Phaeophyta (algas pardas). ${ }^{5,12-18}$

No presente trabalho é descrito o isolamento de uma nova substância sulfatada a partir da alga vermelha Bostrychia tenella, cujo gênero Bostrychia Montagne é o terceiro em termos de ocorrência na família Rhodomelaceae. Há um número considerável de estudos morfológicos, mas, com relação ao metabolismo secundário, há uma escassez de estudos quanto aos constituintes químicos de suas espécies. ${ }^{19}$

\section{RESULTADOS E DISCUSSÃO}

A partir dos estudos químicos realizados, foi possível identificar e elucidar as estruturas de duas substâncias contidas nas frações 17 e 18, obtidas do fracionamento do extrato bruto da espécie B. tenella em coluna Sephadex LH20.

A análise dos espectros de RMN de ${ }^{1} \mathrm{H}$, de ${ }^{13} \mathrm{C}$, DEPT $135^{\circ} \mathrm{e}$ bidimensionais HMQC e COSY ${ }^{1} \mathrm{H}-{ }^{1} \mathrm{H}$ da substância 1 indicou a presença de hidrogênios característicos de anel benzênico e de metileno. Os hidrogênios aromáticos foram observados em $\delta_{\mathrm{H}} 7,20$ e 7,10 ppm; ambos dubletos com constantes de acoplamento de 8,6 $\mathrm{Hz}$, característica de hidrogênios em posição orto; enquanto que o dubleto em $\delta_{\mathrm{H}} 4,43 \mathrm{ppm}(J=5,5 \mathrm{~Hz})$ indicou ser característico de hidrogênio de metileno carbinólico. O singleto largo em $\delta_{\mathrm{H}} 5,13$ ppm correspondeu ao sinal de deslocamento químico do hidrogênio do substituinte hidroxílico. Os dados de $\mathrm{RMN}$ de ${ }^{13} \mathrm{C}$ permitiram confirmar os sinais característicos de anel benzênico, como em $\delta_{C} 152,2$ ppm atribuído ao C-1 e os sinais em $\delta_{C} 120,0$ e 126,9 ppm, atribuídos aos carbonos 2,6 e 3,5, respectivamente. O sinal referente ao carbono metilênico foi observado em $\delta_{C} 62,5 \mathrm{ppm}$. O carbono quaternário aromático correspondente à posição 4 foi observado em $\delta_{\mathrm{C}} 137,5 \mathrm{ppm}$.

Com os dados obtidos por meio das análises dos espectros de $\mathrm{RMN}$ de ${ }^{1} \mathrm{H}$ e de ${ }^{13} \mathrm{C}$ foi possível concluir que o anel aromático era dissubstituído nas posições 1 e 4 . Em uma dessas posições foi estabelecida a presença de um grupo metileno ligado a um grupo hidroxílico, mas, com relação à outra posição, restava determinar 
qual era o grupo substituinte. Por meio da análise do espectro de absorção na região no infravermelho da substância 1 pôde-se notar a presença de sinal referente a grupo sulfato, uma vez que a ocorrência de ésteres de sulfatos orgânicos é indicada por duas bandas intensas na região de $1250-1210 \mathrm{~cm}^{-1}$. A literatura descreve ainda para compostos do tipo $\mathrm{CH}_{3} \mathrm{C}_{6} \mathrm{H}_{4} \mathrm{OSO}_{3} \mathrm{~K}$ a presença de bandas intensas em 1268 e $1233 \mathrm{~cm}^{-1}$, além de uma banda em $800 \mathrm{~cm}^{-1}$, corroborando com os dados observados no espectro no IV para a substância 1 (bandas em 1266 e 1237 cm$^{-1}$, além da banda em 799 $\left.\mathrm{cm}^{-1}\right){ }^{20-22}$ Aliada a esta informação, na literatura é mencionada a ocorrência de estruturas fenólicas similares, contendo grupos sulfatos diretamente ligados ao anel aromático e que exibem um deslocamento químico em $\delta_{\mathrm{C}} 155,1 \mathrm{ppm} .{ }^{18}$ Como a substância 1 possui um carbono com deslocamento químico em $\delta_{\mathrm{C}} 152,2 \mathrm{ppm}$, podese inferir que este está diretamente ligado ao grupo sulfato, confirmando a estrutura de $\mathbf{1}$.

Os espectros de HMQC mostram correlações entre os hidrogênios H-2 e H-6 em $\delta_{\mathrm{H}}$ 7,10 ppm com os carbonos C-2 e C-6, com deslocamento químico em $\delta_{\mathrm{C}} 120,0$ ppm; enquanto que os hidrogênios H-3 e H-5 em $\delta_{\mathrm{H}}$ 7,20 ppm são correlacionados com os carbonos C-3 e C-5, com deslocamento químico em $\delta_{\mathrm{C}} 126,9$ ppm. O dubleto em $\delta_{\mathrm{H}} 4,43 \mathrm{ppm}$, referente aos hidrogênios do carbono metilênico, está correlacionado com o sinal em $\delta_{\mathrm{C}} 62,5 \mathrm{ppm}$, ou seja, o carbono carbinólico C- $\alpha$.

O espectro no infravermelho ainda exibiu bandas de absorção em $3375 \mathrm{~cm}^{-1}$ característica de estiramento $\mathrm{OH}$ de grupo hidroxílico associado, duas bandas de intensidade forte em 1266 e $1237 \mathrm{~cm}^{-1}$, característica de estiramento de grupo $\mathrm{SO}_{3}$ e ainda uma banda de absorção em $799 \mathrm{~cm}^{-1}$, característica de deformações axiais do sistema S-O-C. ${ }^{20}$ Os estiramentos relativos à ligação $\mathrm{C}=\mathrm{C}$ do anel aromático são responsáveis pelas bandas de absorção em 1612, 1590 e $1508 \mathrm{~cm}^{-1}$. A substância 1 apresenta fórmula molecular $\mathrm{C}_{7} \mathrm{H}_{7} \mathrm{O}_{5} \mathrm{~S}$, determinada pela análise do espectro de massas via electrospray (ESMS) no modo negativo, onde se pode identificar o íon M-H em 202.9981 u.m.a.. De acordo com a estrutura proposta de massa molecular experimental 202.9981 u.m.a (calculada de 203.00197 u.m.a.), foi confirmada a fórmula molecular para a substância $\mathbf{1}$.

A substância 2, de acordo com os dados obtidos por meio das análises dos espectros de RMN de ${ }^{1} \mathrm{H}$, de ${ }^{13} \mathrm{C}$, DEPT $135^{\circ} \mathrm{e}$ bidimensionais HMQC e COSY ${ }^{1} \mathrm{H}-{ }^{1} \mathrm{H}$ foi identificada como sendo o álcool 1-metoxifeniletílico, previamente obtido por processos sintéticos. ${ }^{23,24}$

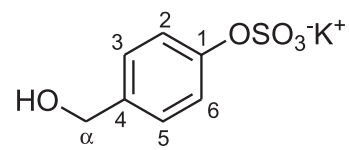

Substância 1

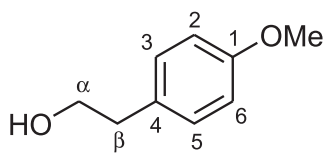

Substância 2
Figura 1. Metabólitos secundários isolados da alga marinha Bostrychia tenella

Sobre a ocorrência destas substâncias, pode-se dizer que metabólitos secundários sulfatados, principalmente quando ligados a anéis aromáticos, são comumente encontrados em organismos vegetais devido à metabolização dos compostos tóxicos a estes. ${ }^{25} \mathrm{~A}$ conjugação com sulfato é um processo muito comum de desintoxicação nos animais. A inclusão de substituinte $\mathrm{SO}_{3}^{-} \mathrm{em}$ uma molécula orgânica ocorre com o objetivo de proporcionar mudança de característica estrutural para facilitar a excreção desta ao meio externo. ${ }^{15}$ Esta hipótese é fortemente alicerçada, levando em consideração várias pesquisas que visam o isolamento de enzimas responsáveis por esta transferência do grupamento químico sulfato para moléculas orgânicas, principalmente as fenólicas. ${ }^{25}$ Assume-se que a formação de ésteres de sulfato ocorre em organismos vegetais via fosfoadenosilfosfossulfato (PAPS). ${ }^{25}$ A enzima fenolsulfatase foi caracterizada e purificada a partir da alga marinha Cystoseira tamariscifolia (Cystoseiraceae, Phaeophytaceae), à qual foi atribuída a capacidade biossintética de originar moléculas orgânicas (fenólicas) sulfatadas nestes organismos. ${ }^{25}$

De acordo com publicações na literatura científica, referentes a compostos semelhantes, não foi relatado nenhum tipo de atividade biológica para esta classe de substâncias. Entretanto, Jensen e Ragan sugerem que sulfatos de baixo peso molecular são precursores fenólicos na alga parda Ascophyllum nodosum, além de outras macroalgas, e participam da formação de compostos encontrados no material orgânico marinho em degradação. ${ }^{16}$

\section{PARTE EXPERIMENTAL}

\section{Procedimentos experimentais gerais}

Os espectros de RMN foram obtidos no espectrômetro Bruker DRX-400, operando em $400 \mathrm{MHz}$ para ${ }^{1} \mathrm{H}$ e $100 \mathrm{MHz}$ para ${ }^{13} \mathrm{C}$, respectivamente, no Departamento de Química da Faculdade de Filosofia, Ciências e Letras de Ribeirão Preto (FFCLRP-USP), SP, Brasil. As amostras analisadas foram solubilizadas em DMSO-d, utilizando-se TMS como padrão interno de análise. Os espectros na região no infravermelho foram obtidos em um espectrofotômetro Perkin Elmer - FTIR série 1420 (Ratio Recordind Infrared Spectrophotometer), utilizando pastilhas de $\mathrm{KBr}$. Os espectros de massas foram obtidos utilizando aparelho de modelo ultrOTOF ${ }_{Q}$ ESI-TOF Mass Spectrometer, Bruker Daltonics, Billerica, MA, EUA. As condições do experimento foram: bomba de infusão, fluxo $300 \mu \mathrm{L} / \mathrm{h}$, fase móvel para a solubilização: $\mathrm{H}_{2} \mathrm{O}: \mathrm{MeOH}(1: 4)$, com modo de detecção negativo para as amostras. Foi utilizada solução de NA-TFA a $10 \mathrm{mg} / \mathrm{mL}$ para calibração interna, sendo que para a calibração externa foi utilizada solução de formiato de sódio, a $10 \mathrm{mM}$. Para cromatografia em coluna foi utilizado Sephadex LH20 (Merck) e para análises em cromatografia em camada delgada comparativa, utilizaram-se placas de Sílica gel $\mathrm{GF}_{254}$, sendo as manchas visualizadas sob irradiações com luz UV e reveladas com iodo ressublimado ou reveladores universais, como $\mathrm{H}_{2} \mathrm{SO}_{4}$ concentrado.

\section{Material algal}

Algas vermelhas da espécie $B$. tenella foram coletadas nas encostas rochosas da Praia de Fortaleza, Ubatuba - SP, Brasil, em outubro de 2004. As algas foram coletadas e identificadas pela Dra. N. S. Yokoya, bióloga pesquisadora do Instituto de Botânica do Estado de São Paulo (IBt) - Brasil e a exsicata foi depositada no herbário do Instituto de Botânica, com número de acesso SP 391087.

\section{Extração e isolamento}

O material foi lavado e transportado em água do mar (com isolamento térmico). Previamente à preparação dos extratos, as algas foram lavadas e secas a temperatura ambiente. O material seco $(74,6 \mathrm{~g})$ foi manualmente fragmentado, pulverizado em nitrogênio líquido e submetido à extração com solvente orgânico $\left(\mathrm{CH}_{2} \mathrm{Cl}_{2}: \mathrm{MeOH}, 2: 1\right)$ a temperatura ambiente, por três vezes em intervalos de $24 \mathrm{~h}$. O solvente foi removido utilizando rotaevaporador, com temperatura de até 40 ${ }^{\circ} \mathrm{C}$. Obteve-se uma massa aproximada de $3,8 \mathrm{~g}$ de extrato bruto.

Cerca de $1,0 \mathrm{~g}$ de extrato foi submetido ao fracionamento 
cromatográfico utilizando-se Sephadex LH20 como suporte, sendo a eluição em metanol. Foram obtidas 32 frações de aproximadamente $15 \mathrm{~mL}$. Deste fracionamento, as frações $17(8,7 \mathrm{mg})$ e 18 $(9,2 \mathrm{mg})$ forneceram as substâncias $\mathbf{1}$ e $\mathbf{2}$, respectivamente.

Substância 1 - 4-(hidroximetil)-benzenossulfonato de potássio: substância amarela amorfa; IV (KBr) $v_{\text {max }} 3375,3309,3044,3010$, 2958, 2920, 2873, 1685, 1648, 1612, 1590, 1508, 1384, 1266, 1237, 1172, 1108, 1063, 1027, 1015, 882, 830, 799, 733, 701, 652, 621 $\mathrm{cm}^{-1}, \mathrm{RMN}$ de ${ }^{1} \mathrm{H}\left(\mathrm{DMSO}-\mathrm{d}_{6}, 400 \mathrm{MHz}\right): \delta 7,20\left(2 \mathrm{H}, \mathrm{d}, J_{3,2}\right.$ e $J_{5,6}=$ $8,6 \mathrm{~Hz}, \mathrm{H}-3$ e H-5), $\delta 7,10\left(2 \mathrm{H}, \mathrm{d}, J_{2,3}\right.$ e $J_{6,5}=8,6 \mathrm{~Hz}, \mathrm{H}-2$ e H-6), $\delta$ $4,43(2 \mathrm{H}, \mathrm{d}, J=5,5, \mathrm{H}-\alpha)$ e $\delta 5,13(1 \mathrm{H}, \mathrm{sl}, \mathrm{OH})$. RMN de ${ }^{13} \mathrm{C}$ (DMSO$\left.\mathrm{d}_{6}, 100 \mathrm{MHz}\right) \delta 152,2(\mathrm{Cq}, \mathrm{C}-1), \delta 137,5(\mathrm{Cq}, \mathrm{C}-4), \delta 126,9(\mathrm{CH}$, C-3 e C-5), $\delta$ 120,0 (CH, C-2 e C-6), $\delta 62,5\left(\mathrm{CH}_{2}, \mathrm{C}-\alpha\right)$, Fórmula molecular $\left(\mathrm{C}_{7} \mathrm{H}_{7} \mathrm{O}_{5} \mathrm{~S}^{-}\right) \mathrm{m} / \mathrm{z} 202.9981$ u.m.a..

Substância 2 - álcool 1-metoxifeniletílico: substância amarela amorfa; IV (KBr) $v_{\max } 3438,3044,3010,2960,2926,2874,1652$, $1613,1509,1452,1383,1170,1110,1062,1051,1019,882,826$, $805 \mathrm{~cm}^{-1}$; RMN de ${ }^{1} \mathrm{H}$ (DMSO-d, $\left.400 \mathrm{MHz}\right): \delta 7,16\left(2 \mathrm{H}, \mathrm{d}, J_{3,2} \mathrm{e}\right.$ $J_{5,6}=8,6 \mathrm{~Hz}, \mathrm{H}-3$ e H-5), $\delta 6,92\left(2 \mathrm{H}, \mathrm{d}, J_{2,3}\right.$ e $J_{6,5}=8,6 \mathrm{~Hz}, \mathrm{H}-2 \mathrm{e} \mathrm{H}-$ $6), \delta 2,51\left(2 \mathrm{H}, \mathrm{t}, J_{\beta, \alpha}=6,8 \mathrm{~Hz}, \mathrm{H}-\beta\right), \delta 3,73\left(2 \mathrm{H}, \mathrm{t}, J_{\alpha, \beta}=6,8 \mathrm{~Hz}, \mathrm{H}-\right.$ $\alpha), \delta 3,80\left(3 \mathrm{H}, \mathrm{s}, \mathrm{OCH}_{3}\right), \delta 2,45(1 \mathrm{H}, \mathrm{s}, \mathrm{OH})$; RMN de ${ }^{13} \mathrm{C}$ (DMSO$\left.\mathrm{d}_{6}, 100 \mathrm{MHz}\right): \delta$ 164,1 (Cq, C-1), $\delta$ 130,0 (Cq, C-4), $\delta$ 129,0 (CH, $\mathrm{C}-3$ e C-5), $\delta$ 120,2 (CH, C-2 e C-6), $\delta 37,5\left(\mathrm{CH}_{2}, \mathrm{C}-\beta\right), \delta 58,9$ $\left(\mathrm{CH}_{2}, \mathrm{C}-\alpha\right), \delta 56,1\left(\mathrm{OCH}_{3}\right)$; Fórmula molecular $\left(\mathrm{C}_{9} \mathrm{H}_{12} \mathrm{O}_{2}\right) \mathrm{m} / \mathrm{z}$ 151.0332 u.m.a.. ${ }^{23}$

\section{AGRADECIMENTOS}

À FAPESP pela bolsa de Iniciação Científica e Projeto BIOprospecTA/Jovem Pesquisador (processo 05/53808-9) concedidos e ao CNPq pelos auxílios concedidos (Projeto Milênio, processo $\mathrm{n}^{\circ}$ 420015/05-1 e Projeto Universal processo 485421/2006-2).

\section{REFERÊNCIAS}

1. Newman, D. J.; Cragg, G. M.; J. Nat. Prod. 2007, 70, 461.

2. Clardy, J.; Walsh, C.; Nature 2004, 432, 829.

3. Kossuga, M. H.; Lira, S. P.; Nascimento, A. M.; Gambardella, M. T. P.; Berlinck, R. G. S.; Torres, Y. R.; Nascimento, G. G. F.; Pimenta, E. F.; Silva, M.; Thiermann, O. H.; Oliva, G.; Tempone, A. G.; Melhem, M. S. C.; Souza, A. O.; Galetti, F. C. S.; Silva, C. L.; Cavalcanti, B.; Pessoa, C. O.; Moraes, M. O.; Hajdu, E.; Peixinho, S.; Rocha, R. M.; Quim. Nova 2007, 30, 1194.

4. Cardozo, K. H. M.; Guaratini, T.; Barros, M. P.; Falcão, V. R.; Tonon, A. P.; Lopes, N. P.; Campos, S.; Torres, M. A.; Souza, A. O.; Colepicolo, P.; Pinto, E.; Comp. Biochem. Physiol., Part C: Toxicol. Pharmacol. 2007, 146, 60.

5. Carvalho, L. R.; Roque, N. F.; Quim. Nova 2000, 23, 757.

6. Duarte, M. E. R.; Cauduro, J. P.; Noseda, D. G.; Noseda, M. D.; Gonçalves, A. G.; Pujol, C. A.; Damonte, E. B.; Cerezo, A. S.; Carbohydr. Res. 2004, $339,335$.

7. Shoeib, N. A.; Bibby, M. C.; Blunden, G.; Linley, P. A.; Swaine, D. J.; Wright, C. W.; Nat. Prod. Commun. 2006, 1, 47.

8. Li, K.; Li, X-M.; Ji, N-Y.; Wang, B-G.; J. Nat. Prod. 2008, 71, 28.

9. Fan, X.; Xu, N. J.; Shi, J. G.; J. Nat. Prod. 2003, 66, 455.

10. Zhao, J.; Fan, X.; Wang, S.; Li, S.; Shang, S.; Yang, Y.; Xu, N.; Yang, L. ; Shi, J.; J. Nat. Prod. 2004, 67, 1032.

11. Ma, M.; Zhao, J.; Wang, S.; Li, S.; Yang, Y.; Shi, J.; Fan, X.; He, L.; J. Nat. Prod. 2007, 70, 337.

12. Glombitza, K. W.; Stoffelen, H.; Murawsky, U.; Bielaczec, J.; Egge, H.; Planta Med. 1974, 25, 107.

13. Kurata, K.; Amiya, T.; Chem. Lett. 1980, 279.

14. Nanteuil, G.; Mastagli, P.; Phytochemistry 1981, 20, 1750.

15. Carvalho, L. R.; Tese de Doutorado, Universidade de São Paulo, Brasil, 1999.

16. Jensen, A.; Ragan, M. A.; Tetrahedron Lett. 1978, 9, 847.

17. Ragan, M. A.; Jensen, A.; Phytochemistry 1979, 18, 261.

18. Glombitza, K. W.; Knoss, W.; Phytochemistry 1992, 31, 279.

19. Duarte, M. E. R.; Noseda, M. D.; Cardoso, M. A.; Túlio, S.; Cerezo, A. S.; Carbohydr. Res. 2002, 337, 1137.

20. Chihara, G.; Chem. Pharm. Bull. 1960, 8, 988.

21. Bellamy, L. J.; The infrared Spectra of Complex Molecules, $3^{\text {rd }}$ ed., Chapman and Hall: London, 1975, vol. 1.

22. Bellamy, L. J. The infrared Spectra of Complex Molecules, $2^{\text {nd }}$ ed., Chapman and Hall: London, 1980, vol. 2.

23. Cho, S-D.; Park, Y-D.; Kim, J-J.; Falck, J. R.; Y, Y-J.; Bull. Korean Chem. Soc. 2004, 25, 407

24. Gomez, C.; Macia, B.; Lillo, V. J.; Yus, M.; Tetrahedron 2006, 62, 9832.

25. Knöss, W.; Glombitza, K-W.; Phytochemistry 1993, 32, 1119. 